\title{
ARTICLE
}

\section{New Criticality Accident Alarm System Detectors at the JAEA Tokai Reprocessing Plant}

\author{
Norio TSUJIMURA* and Tadayoshi YOSHIDA \\ Nuclear Fuel Cycle Engineering Laboratories, Japan Atomic Energy Agency \\ 4-33, Muramatsu, Tokai-mura, Naka-gun, Ibaraki-ken, 319-1194, Japan
}

\begin{abstract}
Criticality Accident $\underline{\text { Alarm }}$ Systems (CAAS) were installed at the NCL Tokai Reprocessing Plant (TRP) in 1984 that are still in service. However, due to the advanced age of the system new replacement CAAS detectors have now been developed. The new detectors are composed of a plastic scintillator coupled with a photomultiplier tube placed into a cadmium-lined polyethylene moderator, and have the feature of being capable of responding to both gammarays and neutrons via capture gamma-rays from $\mathrm{Cd}(n, \gamma)$ reactions. Prototype detectors were built and then operationally tested using critical bursts in a pulsed reactor. The test results demonstrated satisfactory performance. The commercial version systems are currently being field-tested at the TRP and will be fully utilized in October 2009.
\end{abstract}

\section{KEYWORDS: criticality accident alarm system, plastic scintillator, cadmium, moderator, pulsed reactor}

\section{Introduction}

In nuclear facilities where fissile materials are being handled in quantities exceeding the minimum critical amounts, elaborate physical and administrative controls are provided to prevent any accidental criticality. Nevertheless, the occurrence of such an accident cannot be entirely ruled out, as demonstrated in a number of accidents around the world over the last sixty years, and most recently at Tokaimura in 1999. ${ }^{1)}$ A Criticality Accident Alarm System (CAAS) is mandatory at any such facilities, and is an area monitoring system intended to reduce the dose received from a criticality accident by means of detection and immediate area evacuation. $^{2,3)}$

The Nuclear Fuel Cycle Engineering Laboratories (NCL) of the Japan Atomic Energy Agency (JAEA) operates the pilot plants for fuel reprocessing and MOX fuel fabrication, and has been using CAASs for at least 40 years, including periods used by its predecessor organization of the Power Reactor and Nuclear Fuel Development Corporation (PNC). The first CAASs using an ionization chamber were voluntarily deployed at a plutonium fuel development facility in 1965 . The second CAASs, designed by the Commissariat à l'Energie Atomique (CEA) in the early$1960 \mathrm{~s}^{4)}$ and manufactured by the Société d'Électronique Industrielle et Nucléaire (SEIN), were selected for use in the plant design of the Tokai Reprocessing Plant (TRP) and then installed in 1973 during the plant construction. The current CAASs, chronologically numbered third-generation, were domestically developed in the 1977-1981 collaboration with Toshiba Corporation. ${ }^{5)}$ The overall system concept of the third-generation system was not drastically changed from that of the second-generation system, but parts of the detectors and the logic system were redesigned and built to achieve greater reliability. By the end of 1990, all the

\footnotetext{
* Corresponding author, Tel.+81-29-282-1111, Fax.+81-29-282-

9534, E-mail; tsujimura.norio@jaea.go.jp
}

systems, both freshly installed or used for replacement, had been integrated into the third system. ${ }^{6)}$ A total of 30 systems are presently being used throughout the NCL site.

Four systems being used at the TRP have been in service since first being installed in 1984. Although they have successfully functioned as evidenced by 25 years operation without any serious troubles including any false alarms, they have approached the end of their useful life, and therefore need to be replaced. For that reason we initiated the development project of a new generic detector which will be used to supersede custom-made neutron detectors installed in specific areas only of the TRP.

This paper provides an overview of the currently-used and newly-developed CAASs. Moreover, the latest results of performance tests conducted using the pulsed reactor are also presented.

\section{Current Criticality Accident Alarm System \\ 1. General}

A typical schematic diagram of the CAAS is provided in Figure 1. The system is constituted of three detector units, a logic unit composed of three two-out-of-three voting units and a relay unit, an alarm unit that initiates an evacuation warning throughout the facility, and power supplies and subsidiary modules.

A group of three identical but independent detector units are deployed close to a probable accident location for detecting the occurrence of a criticality accident. If the radiation level where a detector is located exceeds the preset threshold value the detector triggers trip signals to the 2-outof-3 unit. Two types of detectors, namely gamma-sensitive and neutron-sensitive, are used.

Trip signals from the detectors are fed to the 2-out-of-3 voting units, which are cross-linked to a total of three detectors. The 2-out-of-3 unit then 'votes' if at least two of the three detectors have provided trip signals within a period 
of $500 \mathrm{~ms}$, and followed by the relay unit that is used to judge whether it is a true emergency with an AND function through a series of relay-contacts from any 'two votes' sent from the three voting units. The dual majority decision logic incorporates a unique feature preventing false alarms due to any single failure of the detectors and/or voting units. ${ }^{6)}$

The alarm unit is activated by the relay unit and then generates both audible and visible evacuation warnings through use of loudspeakers and flashing lights situated throughout the facility. For the alarm activation redundancy, both the relay and the alarm units include identical electrically-isolated circuits. All the power modules supplying the detectors and the control panel are also duplicated.

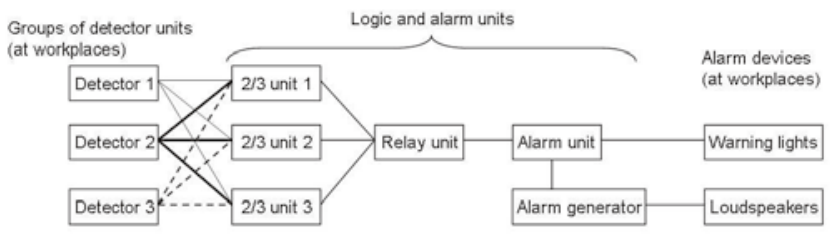

Fig. 1 A block diagram of the criticality accident alarm system.

The 2-out-of-3 voting unit can accommodate up to 12 detector units ( $=4$ systems)

\section{Gamma Detector}

While 87 gamma-sensitive detector units, constituting 29 systems, are being operational at the NCL site, nine detector units are currently being used in three areas at the TRP.

The detector unit (Toshiba RD120) consists of a gammaray detector and related electronics circuits. The unit uses a cylindrical plastic scintillator, $3.8 \mathrm{~cm}$ in diameter and $5.1 \mathrm{~cm}$ long, coupled to a photomultiplier tube operated in current mode. The anode current of the photomultiplier is converted into a voltage by an operational amplifier. The signal is proportional to gamma-ray dose rates, and normally indicates $\sim 1 \mathrm{~V}$ as a constant 'live' background. Any incremental output of $0.72 \mathrm{~V}$ corresponds to a dose rate of $1.0 \mathrm{mGy} \mathrm{h}^{-1}$. A comparator provides the trip signal when the amplifier output voltage exceeds the preset value, which is usually set at 1 to $3.6 \mathrm{mGy} \mathrm{h}^{-1}$ in tissue absorbed dose rate, depending on the distance and intervening shields.

Extensive performance tests carried out in the 1980s proved that the detector units functioned correctly in both the pulse radiation field of a pulse reactor and an extremely intense radiation field $\left(>10 \mathrm{kGy} \mathrm{h}^{-1}\right.$ ) of a strong ${ }^{60} \mathrm{Co}$ gamma source. In addition, as recently reported by the authors ${ }^{7)}$, while the detector responds primarily to gamma-rays, it is also slightly responsive to neutrons due to the capture gamma-rays produced from neutrons interacting with the structure of the detector.

\section{Neutron Detector}

Three neutron-sensitive detector units have been used in a particular area of the TRP to monitor a plutonium nitrate solution vessel housed in a thick steel walled cell that precludes any gamma-rays being detected.
The detector unit (Toshiba RD624) uses a silicon surface barrier detector (SSD) with $\sim 90 \%$ enriched ${ }^{235} \mathrm{U}$ convertor that is surrounded with a $9 \mathrm{~cm} \times 9 \mathrm{~cm} \times 9 \mathrm{~cm}$ acrylic moderator to increase detection efficiency of fast neutrons. The SSD output signals produced by fission fragments are amplified, and then fed into a digital integrator/counter circuit, which actuates a trip signal driver circuit when the count increases to more than 5 within 5 seconds, corresponding to a neutron tissue absorbed dose rate of $\sim 2$ $\mathrm{mGy} \mathrm{h}^{-1}{ }^{8)}$

The large Q-value of fission reactions enables the unit to discriminate between gamma-rays, alpha-particles from ${ }^{235} \mathrm{U}$ and electrical noise to an excellent degree. However, a number of problems exists in terms of their maintainability. The major problem is that the diode detector needs to be changed every 5 -years due to radiation damage caused by spontaneous alpha-particles from ${ }^{235} \mathrm{U}$. Furthermore, the detector unit is of a very outdated design, and thereby the spare components are very unit-specific, i.e., not interchangeable with those of a gamma detector unit (accounting for the majority of the parts inventory). In addition, use of special nuclear materials has incurred administrative cumbersomeness.

\section{New Criticality Accident Alarm System 1. Design Strategy}

As mentioned in the Introduction, more than 20 years have passed since the CAAS was first installed at the TRP in 1984, necessitating its overall replacement. Our consensus on that replacement was that although the gamma detectors still fit their purpose, the neutron detectors require a drastic change in design because of the above problems. The development project of a new detector that would supersede the neutron detector was initiated in collaboration with Toshiba Corporation for that purpose.

The design goals of the new detector were: (1) to incorporate an off-the-shelf neutron-sensitive detector; (2) to ensure better maintainability for the CAAS operational program, including standardization of all the hardware and maintenance procedures; and (3) to keep both cost and risk in the initial development as low as possible. In addition, a detector of simple and robust construction was a key requirement in achieving high reliability. Needless to say, it should avoid incorporating any special nuclear materials as part of the detection system.

To achieve all the above mentioned goals, the design strategy with the new detector was determined to modify the existing gamma detectors to be neutron-sensitive by incorporating additive materials that could act as a neutronto-gamma converter. That modification would add the capability to detecting neutrons to a gamma detector unit without degrading its overall performance which has been demonstrated for many years. Furthermore, this approach that makes the most of a proven detector appeared to be advantageous to satisfy the safety regulators, who are usually cautious about approving any new technology. 


\section{Description of prototype detector}

Figure 2 gives a cross-sectional schematic view of the final prototype detector unit. ${ }^{9)}$ A cylindrical plastic scintillator, identical to that used in the gamma detector unit, is surrounded with a cylindrical polyethylene moderator incorporating dual layers of cadmium (Cd). Cadmium was selected as an additive neutron-to-gamma-rays converter material because of its high neutron-capture cross-section and availability at low cost. The total thickness of the polyethylene moderator is $5 \mathrm{~cm}$, with each of the Cd layers being $1 \mathrm{~mm}$ thick. The optimum configuration was determined using the Monte Carlo calculations to be capable of responding proportionally to total absorbed doses, regardless of whether it was exposed to neutrons, gammarays or a combination of both. More details on the design process are described elsewhere. ${ }^{10)}$ A simplified explanation of the detection mechanism is that slow and fast neutrons get moderated and then absorbed by the respective outer and inner $\mathrm{Cd}$ layers, thus providing prompt gamma-rays (from the neutron capture of the ${ }^{113} \mathrm{Cd}$ ) that are detectable by the central scintillator.

Two prototype detector units were built and then laboratory-tested with respect to the dose (rate) linearity, energy and angular dependences, a long-term background stability, etc. The test results demonstrated satisfactory performance commonly required of a CAAS detector. The results of the laboratory testing are also reported elsewhere. $^{10)}$

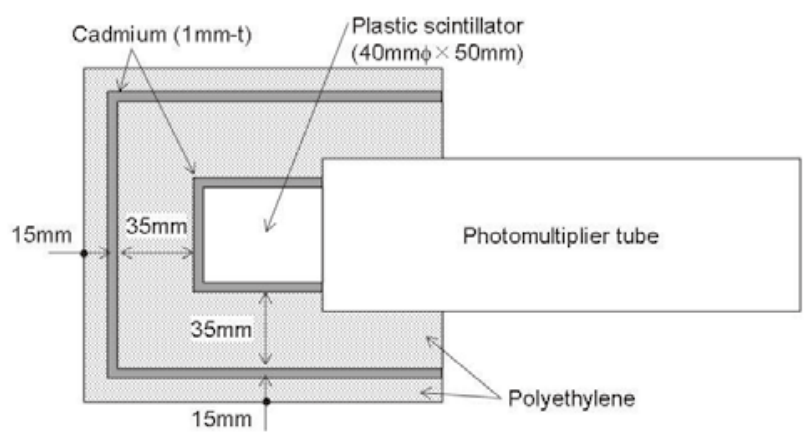

Fig. 2 A cross-sectional schematic view of the prototype detector

\section{Operational Testing at the TRACY 1. Description of TRACY}

An important factor was to confirm that the detector design modifications had not adversely changed the characteristics of the detector. Specifically, the detector response to pulsed radiations with a short duration and high dose rate was of special concern. Tests were therefore carried out at TRACY (Transient Experiment Criticality Facility) at the Nuclear Science Research Institute of the JAEA. TRACY is a pulse-type reactor that uses $\sim 10 \%$ enriched uranyl nitrate solution as fuel, the components of which are close to the solutions to be generally processed in reprocessing plants. ${ }^{11)}$ Rapidly withdrawing the transient rods, which are made of natural boron carbide, results in a quick momentary critical configuration. The maximum integrated power can be up to $32 \mathrm{MJ}$, which is equal to $1 \times 10^{18}$ fissions.

\section{Test Methods}

Figure 3 shows the experimental setup. The prototype detector unit was placed at the locations A or B that can be seen in Fig. 3. Position A is on the floor of a room directly above the reactor room and is $12 \mathrm{~m}$ from the center of the reactor core with $190 \mathrm{~cm}$ of concrete between them. Position $\mathrm{B}$ is $6 \mathrm{~m}$ away from the reactor core on the floor of the reactor room. The detector output signals, both analog and trip, get transmitted to a control room by twisted shielded pair cables and then recorded with a high speed digital recorder, Yokogawa Model AR 4800. The output signal of the reactor power monitor, a micro fission chamber precalibrated to the number of fissions, was also simultaneously recorded. Thermoluminescence dosemeters of $\mathrm{CaSO}_{4}: \mathrm{Tm}$ were also placed near each detector to measure the delivered doses.

All the tests were performed as "parasitic" without disrupting any prescheduled reactor operations. Therefore, the sizes and durations of the power bursts were not tailored for testing of the detector.

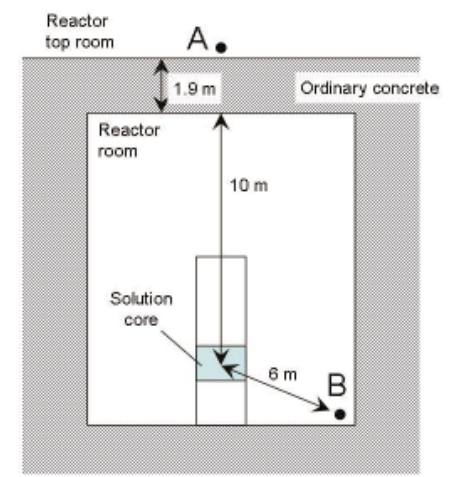

Fig. 3 Experimental setup at TRACY (not to scale)

\section{Results and Discussion}

Table 1 summarizes the reactor operating conditions and the results of the detector tests. Run numbers R351, R395 and R401 involved pulse response tests, while R402 an overload test. The pulses ranged from $2 \times 10^{17}$ to $5 \times 10^{17}$ fissions, with the Full Width Half Maximum (FWHM) duration of the pulses ranging from 12 to $39 \mathrm{~ms}$. The pulses tend to broaden as the peak rate decreases.

Figure 4 provides a graph of the analog and trip signals from the detector unit in position A caused by the shortest duration pulse of R351. The abscissa is the elapsed time after withdrawal of the transient rod. Also depicted is the output, normalized to unity at the peak, of the micro fission chamber for the sake of comparison. The voltage of the analog signal started to rapidly rise at $130 \mathrm{~ms}$ from the background baseline $(\sim 1 \mathrm{~V})$, without any delay with that of the micro fission chamber, and was then saturated. The change in the analog signal switched the trip signal from a normal status $(14 \mathrm{~V})$ to an alarm status $(1 \mathrm{~V})$ during a time interval from $130 \mathrm{~ms}$ to $170 \mathrm{~ms}$ when the analog signal exceeded the trip level of $1.56 \mathrm{~V}$, corresponding to the 0.87 $\mathrm{mGy} \mathrm{h}^{-1}$, preset prior to the test. This demonstrated that the detector unit was saturated but not blinded, and certainly activated the logic unit.

The overload test, conducted in the reactor room, revealed that the detector unit functioned properly without any indication of malfunction during the burst, and then continued to operate properly after the burst. 
Thermoluminescent dosemeters placed near the detector unit indicated an integrated gamma dose of $0.08 \mathrm{~Gy}$. This gave a total dose (gamma + neutron) of 0.09 Gy when using the neutron-to-gamma dose ratio of 0.1 from previous measurements made by Murazaki et al. ${ }^{12)}$ Assuming that the entire dose was delivered in twice the FWHM time interval, the dose rate was estimated to be $>3.7 \mathrm{kGy} \mathrm{h}^{-1}$, satisfying the IEC's dose level requirements of at least $1 \mathrm{kGy} \mathrm{h}^{-1}$ for overload tests. $^{13)}$

Testing at TRACY verified that the new detector units responded correctly to actual transient criticality.

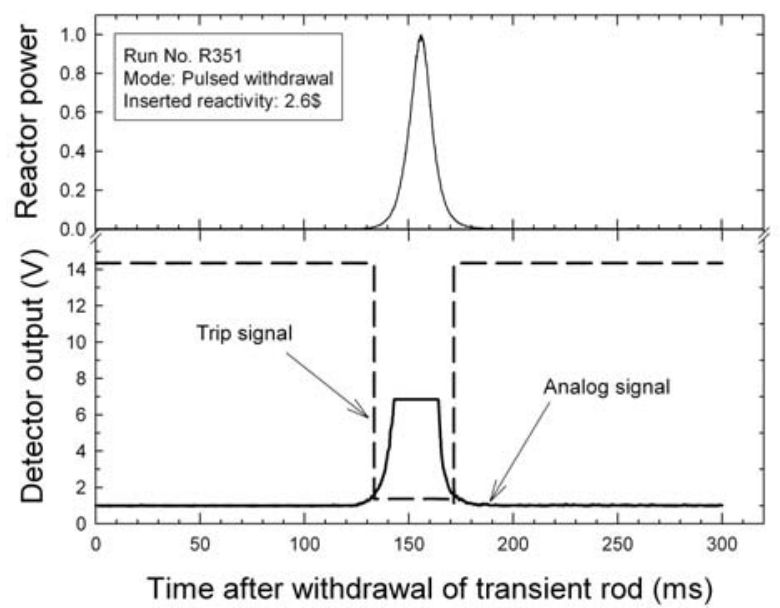

Fig. 4 Detector outputs as a function of time for the R351 TRACY operation. Upper figure shows the relative power measured with the reactor power monitor.

Table 1 TRACY operating conditions and the results of detector test

\begin{tabular}{ccccc}
\hline Run No. & $\begin{array}{c}\text { Peak } \\
\text { power } \\
(\mathrm{MW})\end{array}$ & $\begin{array}{c}\text { Pulse } \\
\text { duration } \\
(\mathrm{ms})\end{array}$ & $\begin{array}{c}\text { Detector } \\
\text { position }\end{array}$ & $\begin{array}{c}\text { Test results } \\
\text { Pass/Fail }\end{array}$ \\
\hline R351 & 1050 & 12 & A & Pass \\
R395 & 220 & 30 & A & Pass \\
R401 & 135 & 39 & A & Pass \\
R402 & 135 & 39 & B & Pass \\
\hline
\end{tabular}

\section{Summary}

In the TRP of the NCL, Criticality Accident Alarm Systems (CAAS) were installed in 1984 and are still in service. However, due to the advanced age of the system new replacement CAAS detectors have now been developed. The new detectors are composed of a plastic scintillator coupled with a photomultiplier tube that is placed into a cadmium-lined polyethylene moderator, and have the feature being capable of responding to both neutron and gammarays in proportion to total absorbed doses of the two types of radiations. And because the new detector unit is essentially a modified version of the existing gamma-sensitive detector unit, the resulting compatibility will contribute to standardization in the necessary maintenance programs.

At the conclusion of the development phase, the prototype detector units were tested using critical bursts from the pulse-type reactor TRACY, and satisfactory performance was demonstrated. The new commercial version systems are currently field-tested at the TRP and will commence operation in October 2009.

\section{Acknowledgements}

We would like to thank the staff members of the Nuclear Fuel Cycle Safety Research Facility (NUCEF), JAEA, for allowing us to make measurements at the TRACY, and in particular Drs E. Aizawa, K. Tonoike and H. Yanagisawa. We also sincerely thank Mr. H. Miyata for his assistance with the measurements.

\section{Reference}

1) T. P. McLaughlin, S. P. Monahan, N. L. Pruvost et al., A Review of Criticality Accidents, 2000 Revision, LA-13638, Los Alamos National Laboratory, (2000).

2) American Nuclear Society, American National Standard for Criticality Accident Alarm Systems, ANSI/ANS-8.3-1996 (1996).

3) Working Group on Nuclear Criticality Safety Data, Nuclear Criticality Safety Handbook, Version 2, JAERI 1340, Japan Atomic Energy Research Institute, (1999), [in Japanese].

4) G. Debrié, J. M. Lavie and M. Planque, "Détection des Accidents de Criticité," Proc. Third Int. Conf. on the Peaceful Uses of Atomic Energy, Vol. 13, 244-252 (1964).

5) M. Kanamori, K. Noda, O. Nakamura, "The Criticality Detection and Alarm System in PNC Tokai Works," Proc. of Int. Seminar on Nuclear Criticality Safety, Tokyo, Japan, Oct. 19-23, 1987, 471-474 (1987).

6) K. Noda, "Development of Criticality Accident Alarm System," PNC Technical Review, 81, 59-62 (1992), [in Japanese].

7) N. Tsujimura, T. Yoshida, "Energy and Angular Responses of the Criticality Accident Detector Using a Plastic Scintillator," J. Nucl. Sci. Tech., 43, 903-907 (2006).

8) N. Tsujimura, T. Yoshida, A. Ishidzuka, "Energy and Angular Responses of Criticality Accident Alarm Systems - Plastic Scintillator and Fission Detector with Moderator," Proc. of the 7th NUCEF Seminar, JAERI-Conf 2004-011, 89-90 (2004), [in Japanese]

9) N. Tsujimura, T. Yoshida, M. Ishii, "Development of Criticality Accident Detector Measuring Neutrons and Gamma-rays," JNC Technical Review, 28, 85-86 (2005), [in Japanese].

10) N. Tsujimura, T. Yoshida, M. Ishii, Development and Characterization of a Criticality Accident Alarm System Detector Sensitive to Neutrons and Gamma Radiations, JAEA Report, Japan Atomic Energy Agency (in preparation).

11) H. Yanagisawa, K. Nakajima, K. Ogawa, et al., "Experiments on Transient Behavior of a Low-enriched Uranyl Nitrate Solution System with TRACY to Study Hypothetical Criticality Accidents in Reprocessing Plants," Proc. 6th Int. Conf. Nuclear Criticality Safety (ICNC), Versailles, France, Sep. 20-24, 1999, Institut de Protection et de Sûreté Nucléaire, Vol. II, 900 (1999).

12) M. Murazaki, K. Tonoike, G. Uchiyama, "Measurement of Neutron Dose under Criticality Accident Conditions at TRACY Using TLDs," J. Nucl. Sci. Tech., 46, 193-203 (2009).

13) International Electrotechnical Commission, Warning Equipment for Criticality Accidents, IEC International Standard 80860, (1987). 